\title{
A design of multifunctional IPTV STB
}

\author{
WU Dongyan, CAO Jiandong, ZHANG Wen, BU Shupo \\ Department of electronics and Communication Engineering \\ Suzhou Institute of Industrial Technology \\ Suzhou,CHINA \\ wudy@siit.cn, caojd@siit.cn, zhangw@siit.cn,busp@siit.cn
}

\begin{abstract}
IPTV is a use of cable television networks, Internet, multimedia, communications and other technologies in one. It provides more information and program for people.STB is the indispensable equipment in IPTV system. This article mainly aims at the existing IPTV STB features, provides a design of IPTV STB which able to accept the terrestrial, satellite and CCTV signal.
\end{abstract}

\section{Keywords- IPTV;STB; PNX8550;Linux}

\section{INTRODUCTION}

With the advent of television technology unceasing development, IPTV has entered into thousands of households. IPTV is a set of cable television networks, Internet, multimedia, communications and other technologies in one, to home users to provide digital television includes a variety of interactive services, new technology. IPTV STB is the core of the IPTV system equipment. However, the existing technology, the media signal receiving and decoding device for targeting specific signaling functions in a single receiver decoding device, can simultaneously accept multiple signal to broadcast. If the user needs to receive terrestrial and satellite television signal, to achieve a variety of signal is received, the user needs to configure more than two set-top box. A STB can drive a plurality of analog or digital TV system in terms of price and performance is attracting the interest of a user's competitive advantage, thus providing a can drive a plurality of analog or digital TV system of the new television network STB has become the field urgently needs to solve the technical problem. Therefore, this paper provides a ground, satellite, CCTV signal transmission and driving a plurality of TV's IPTV STB.

\section{IPTV}

IPTV using computer, or set-top box (STB) and TV to complete video on demand program is received, and surf the Internet video broadcasting function. IPTV STB through standard decoding algorithm including MPEG4, H.264, AVS, VC-1 and others, so that the video may have close to DVD reception effect. In the future, the video service such as the Internet video broadcast, remote true video on demand, program production, have a strong advantage.

Internet Protocol television (IPTV) is a system through which television services are delivered using the Internet protocol suite over a packet-switched network such as the Internet, instead of being delivered through traditional terrestrial, satellite signal,and cable television formats[1] [2].

\section{STB}

STB is connected to a television and an external signal source device. It can be compressed digital signals into television content, and on the TV show. Signal can be derived from the cable, satellite antenna, broadband network and terrestrial broadcasting. The set-top box receives content except for analog TV can provide images, sounds, and more is capable of receiving content data, includes an electronic program guide, the Internet webpage, subtitles and so on. Users can use the existing TV to watch digital TV programs, and through the network of interactive digital entertainment, education and commercial activities[3] [4].

\section{DESIGN SCHEME}

\section{A. structure}

IPTV STB includes Tuners, SoC, Video Encoder, audio $\mathrm{D} / \mathrm{A}$ converter, the infrared receiving processor and distribution module. Among them, SoC (PNX8550) is the core of the system processing section, and embedded Linux operating system. The structure is shown as Fig.1

1) Tuners

DMB-TH is for receiving terrestrial television;

DVB-C is for receiving cable television;

DVB-S is for receiving satellite television;

2) $\mathrm{SoC}(\mathrm{PNX8550)}$

The PNX8550 is a highly integrated media processor for building digital TV receivers with advanced TV and connectivity features. On a single chip, the PNX8550 integrates conditional access, MPEG-2 transport stream demux, video decoding, high-quality video enhancement, audio decode and mixing, graphics generation, image composition, and display.

As a highly integrated media processor, PNX8550 chip comprises three processor cores: a $250 \mathrm{MHz}$ MIPS32 processor, two $240 \mathrm{MHz}$ VLIW multimedia processor.MIPS32 running the Linux operating system, network communication, file system, storage management non real time tasks; multimedia processor for audio processing, such as: MPEG4, H.264 codec.At the same time, chip and hardware module to perform some core video operation, such as a hardware MPEG2 decoder, video scaling of the MBS (Memory Based Scaler), responsible for the image synthesis, processing and the output of the QVCP (Quality Video Composition Processor).

PNX8550 includes a series of function expansion interface, where a PCI-HOST component, through PCI bus 
expansion more functions, making the product is easy to adapt to future needs, such as serial ATA memory.

PNX8550 family entertainment engine two basic chip edition now available.Nexperia PNX8551 home entertainment system chip with enhanced properties, including portable media and the MP3 media format for flash media storage connection and support.PNX8852 is PNX8550 family entertainment engine alternative chip, for television manufacturers to provide a low cost solution for mid-range, mid-range television from analog to digital conversion.

3) Video Encoder

It is mainly for the input signal is converted into a television video signal can accept.

\section{4) Audio D/A converter}

It is mainly to the completion of the audio signal converter.

5) Infrared Receiver STB.

It mainly deal with infrared signal to control the IPTV

\section{6) Distribution Module}

It mainly completes the output television signal distribution, select the desired signal to the TV.

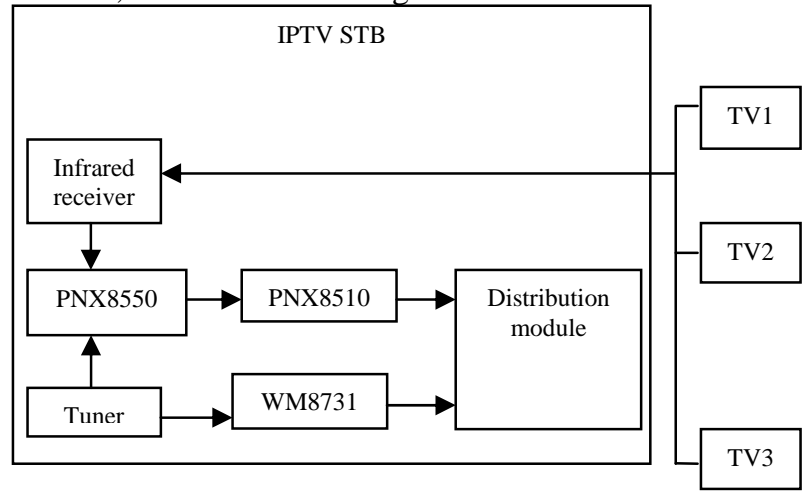

Figure 1. Structures of IPTV STB

\section{7) Inter-Frame Multiplexing}

Because FLASH animation interface motion is continuous, in the previous frame on the basis of need only update the frame buffer area, you can get a new image, reduce hardware resources.

But in order to reduce the need for rendering the calculation part, must adopt effective inter-frame multiplexing technique, provide playback speed.Before and after the two frame with different shapes by PlaceObject2 and RemoveObject2 to control.

When the execution of each frame of these two types of labels, will be the layer of the display area and the area defined by the intersection of the film the rectangular area joined in clipbounds record, if the layers in the previous frame also appeared, the layer of old area should also be recorded.Then the frame of all clipbounds records were combined, as far as possible into each other is not overlapped rectangular region, the inter-frame multiplexing technique improves the decoding efficiency.

\section{OPERATIONAL PRINCIPLES}

Tuner receives the RF signal for demodulation, demodulation of TS flow signal. TS flow signal is input to the PNX8550.PNX8550 will be sent from network streaming media after decoding, video stream and audio solution program stream. Through the PNX8550 internal software, solutions to check ITU56 standard video data, the data is sent to a video encoder (PNX 8510), a simulation with the video signal, S-VHS signal and the video signal output. At the same time the audio program stream decompression, work out digital audio signal, the audio signal to the audio D/A converter (WM8731L), after D/A conversion, the analog audio stereo output [5].

Through the LAN/WAN card, HomePNA2.0 card for receiving from a TV1 to a terrestrial broadcast signal remote control (R/C) signal, $\mathrm{R} / \mathrm{C}$ signal is input into PNX8550.PNX8550 from the input R/C signal to identify the corresponding TV1 ID. Control allocation module enables the LNB is received and processed by the PNX8550 broadcast signal can be transmitted to the TV1.

The infrared receiving processor receives the infrared remote controller to control signal, and after treatment sent to PNX8550.Distribution module according to the control of the PNX8550 signal are respectively assigned to each television broadcast signal. Distribution module according to the control of the PNX8550 through LAN, WAN or RF cable broadcasting signals corresponding to the TV1, thereby ground broadcast signal can be transmitted to the TV1. When users watch the display the specific ground radio and TV1, TV2 and TV3 satellite, cable television.

\section{SOFTWARE}

\section{A. .Linux}

The embedded Linux system is the biggest advantage is: open source code, there are a large number of free development tools and software applications available, without paying royalties; have huge development groups, exchange of technology, software development and maintenance cost is low; the stability of the system, the kernel is concise, operation needs less resources, high cost performance.

The operating system is responsible for set top box system of all software and hardware resource allocation, scheduling, control and coordinate the various instructions, can pass through a loading and unloading systems of certain modules to achieve the required functions.STB software structure, with the Linux operating system as the core, down through the various drivers. The software system is shown as Fig.2.

For the hardware system, the deployment of a variety of hardware resources, to be loaded with audio and video related applications and finish the other task application. Because the system of multimedia microprocessor PNX8550 itself not only have embedded RISC operation system software and application software. In the introduction of Linux operating system and the necessary video and the auxiliary interface driver, is a complete interactive video network terminal operation environment. 
IPTV STB due to the features of complex, high performance requirements, the software generally to a realtime operating system (Embedded Systems) as the core, according to the hardware and system function to be expanded. Linux is open source software; anyone can modify it, or use it to develop their products by themselves. The Linux system can be customized, system kernel now can do very little. With an operating system and graphical interface procedures can be less than $1 \mathrm{MB}$ core, but also stable.

A scalable software platform system, is the development of future embedded equipment product excellent resources, many Linux fans can give Linux developers powerful technical support. Therefore selecting Linux as the embedded operating system is very promising. Embedded Linux embedded target system according to the requirements of design, by a small kernel and some may need to be trimmed system module, the system is generally not more than a few[6].

At the bottom of all kinds of hardware components, including MPEG system and processing unit, audio / video / graphics processing unit, Flash memory, serial, parallel port, the smart card interface, network communication components, the driver directly access these components; for the upper software for the use of the equipment, in the driver layer a driver management, it from the peripheral interface, MPEG system processing, audio / video / graphics, communication aspects, management of the underlying physical drive interface, thereby facilitating high-level functions, also reduce CPU burden; another layer is real-time operating system kernel, the software is the core of the system, it performs the task scheduling, real-time monitoring, resource allocation; Linux system control layer and a library program: system control layer is provided on the audio and video data control, user access interface, communication, channel selection and other advanced features, but also supports multiple applications for these functions sharing and reuse, system interaction is here control and implementation of the database program, provides a system of video, audio and other image processing functions and programs; the highest level for Use layer, processing various application function [7].

\begin{tabular}{|c|c|c|}
\hline Play software & Web & Others \\
\hline Middleware \\
\hline Linux operating system \\
\hline Driver \\
\hline Hardware \\
\hline
\end{tabular}

Figure 2. Software system of IPTV STB

\section{B. Middleware}

Middleware is computer software that connects software components and applications. The software consists of a set of enabling services that allow multiple processes running on one or more machines to interact across a network. This technology evolved to provide for interoperability in support of the move to coherent distributed architectures, which are used most often to support and simplify complex, distributed applications. It includes web servers, transaction monitors, and messaging software [8].

\section{1) Personaljava}

Personal Java was a Java edition for mobile and embedded systems based on Java 1.1.8 . It has been superseded by the CDC's Personal Profile which is not widely deployed [9].

2) Java TV API

Java TV API is a the development of research on the Java platform based on the development of products by Sun company. It is Personal Java development environment expansion, allowing programmers to generate TV center programs and services. It also provides the digital set-top box unique to many of the features of the control. Java TV API is directed to the digital television application specific design, these include: (1) audio and video stream control; (2) the broadcast data acquisition; (3) business information data access; (4) TS stream decoding And a demodulation control; (5) screen graphics processing.

Java TV API for interactive TV design, with media synchronization and application software module function. Media synchronization is the instigation of television program and a background video audio synchronization; application software modular be interactive application software and traditional TV programs and harmonious coexistence.

Java TV API and its potential of individual Java platform for digital interactive television services provided the ideal development and application platform, it will become the TCI digital set-top boxes standard application programming environment, and specific uses which kinds of real-time operating system.

\section{CONCLUSIONS}

According to the existing STB deficiency, this paper provided a receiving terrestrial, cable and satellite digital broadcasting and featuring IPTV STB, has strong function and low cost etc.

\section{ACKNOWLEDGMENT}

The authors thank the science and technology program of Suzhou ( project number:SYG201248).

\section{REFERENCES}

[1] Information on http://en.wikipedia.org/wiki/IPTV

[2] Information on http://baike.baidu.com/view/4425.htm

[3] Information on http://baike.baidu.com/view/16791.htm

[4] Information on http://en.wikipedia.org/wiki/Set-top_box

[5] Dongyan Wu,Shupo Bu. "A New Design of IPTV STB,”Journal of Soochow University(Engineering Science Edition),Vol.32(1) ,Jan. 2012, pp.7-9.

[6] SUN Hui,LI Dan.”Design of High Definition Set Top Box Based on Interactive Video Network” Video Engineering,Vol.34(5), Mar.2010, pp.50-53.

[7] WANG Guo-feng, ZOU Jian-hua.”Design of Multimedia Set-TopBox and its Key Technologies" Video Engineering,Vol.28(4), Mar.2004, pp.43-44.

[8] Information on http://baike.baidu.com/view/23710.htm

[9] Information on http://en.wikipedia.org/wiki/PersonalJava 